\title{
Mechanical Commissioning of the ATLAS Barrel Toroid Magnet
}

\author{
A. Foussat, H. H. J. ten Kate, A. Dudarev, H. Bajas, P. Védrine, C. Berriaud, Z. Sun, and M. Sorbi
}

\begin{abstract}
ATLAS is a general-purpose detector designed to run at the highest luminosity at the CERN Large Hadron Collider. Its features include the $4 \mathrm{~T}$ Barrel Toroid magnet, the largest superconducting magnet $(25 \mathrm{~m}$ long, $20 \mathrm{~m}$ diameter) that provides the magnetic field for the ATLAS muon spectrometer. The coils integrated at CERN, were tested individually at maximum current of $22 \mathrm{kA}$ in 2005 . Following the mechanical assembly of the Barrel Toroid in the ATLAS underground cavern, the test of the full Barrel Toroid was performed in October 2006. Further tests are foreseen at the end $\mathbf{2 0 0 7}$ when the system will include the two End Cap Toroids (ECT). The paper gives an overview of the good mechanical test results achieved in comparison with model predictions and the experience gained in the mechanical behavior of the ATLAS Toroidal coils is discussed.
\end{abstract}

Index Terms-ATLAS, mechanical tests, superconducting coils, toroidal field coil.

\section{INTRODUCTION}

$\mathbf{T}$ HE barrel toroid magnet is part of the system of ATLAS (Apparatus Toroidal Large Aperture Spectrometer) large air-core toroid magnets that provide the magnetic field needed for the ATLAS muon spectrometers [1]. The Barrel Toroid (BT), see Fig. 1, is composed of eight superconducting coils each housing two double pancakes with in total 120 turns of Aluminum stabilized $\mathrm{NbTi} / \mathrm{Cu}$ superconductor carrying up to 20.5 $\mathrm{kA}$ in nominal operation at $4.6 \mathrm{~K}$. Each double pancake is inserted and glued in an A15083 casing under pre-stress to limit interfacial shear stress to $10 \mathrm{MPa}$. The aluminum casing provides a stiff mechanical structure to the coil. The cold mass is housed in a stainless steel $304 \mathrm{~L}$ vacuum vessel. The Barrel Toroid structure ( $22 \mathrm{~m}$ diameter and $25 \mathrm{~m}$ length) is assembled symmetrically around the colliding beam axis. The eight coils are interlinked and supported by an aluminum alloy warm structure unique in size, design and manufacturing. This novel structure is challenging as it should guarantee reliable toroid operation during the next 15 years.

The warm structure supports not only the eight coils but also about 340 tons of muon detectors, services and access structures

Manuscript received August 28, 2007. This work is funded by the ATLAS Collaboration, presently about 1900 scientific authors from 164 institutes in 35 countries worldwide, and is supported in part by CERN.

A. Foussat, H. H. J. ten Kate, A. Dudarev and H. Bajas are with the European Center for Nuclear Research-CERN 1211, Geneva CH, Switzerland (e-mail: Arnaud.Foussat@ cern.ch).

P. Védrine, C. Berriaud and Z. Sun are with CEA, Gif-sur-Yvette, France.

M. Sorbi is with INFN-Laboratorio LASA, Milano, Italy.

Color versions of one or more of the figures in this paper are available online at http://ieeexplore.ieee.org.

Digital Object Identifier 10.1109/TASC.2008.920810

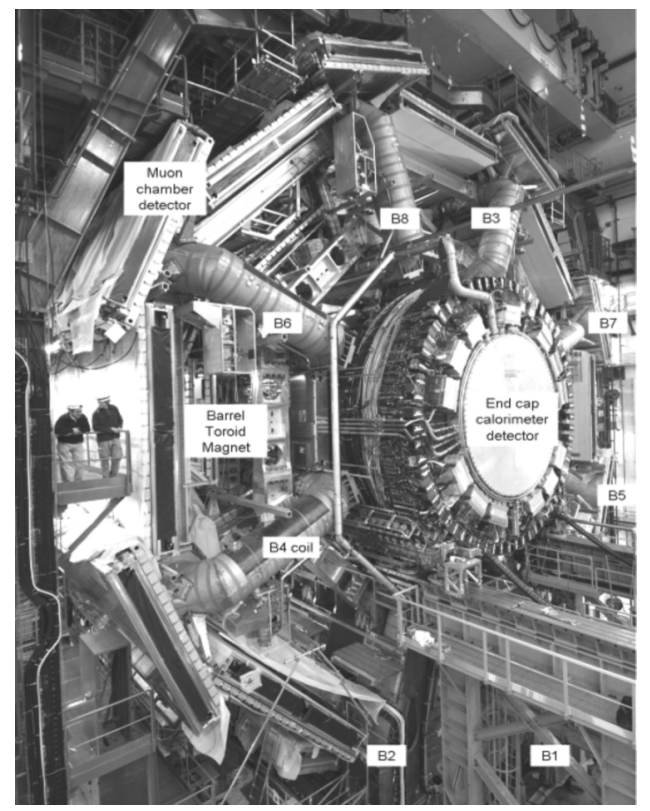

Fig. 1. Overall view of ATLAS barrel toroid magnet installed in the underground ATLAS cavern at CERN.

in ATLAS which constitutes a total mass of 1400 tons. During excitation of the Barrel Toroid, each coil is reacting in-plane centering magnetic forces of maximal $15 \mathrm{MN}$. Due to the size of the individual Barrel Toroid coils as well as the infeasibility of repairs once installed, a representative test of the single coils on surface at nominal current of $20.5 \mathrm{kA}$ is mandatory.

The comparable electromagnetic forces were generated by testing the coil in close contact with a magnetic mirror. These tests in years 2004-2005 allowed qualifying both magnet design choices and the mechanical operation of the cold mass suspension system up to $1800 \mathrm{kN}$ in a single tie rod [2]. The mechanical assembly of the Barrel Toroid started in the underground ATLAS cavern at the end of 2004 and lasted up to autumn 2005 [3]. The mechanical installation was followed by the completion of the electrical system, cryogenic connections and the Magnet Control system. Finally in autumn 2006, the completed Barrel Toroid was gradually powered up to the nominal current of 20.5 kA.

\section{OPERATIONAL LOADS OF THE BARREL TOROID COILS}

\section{A. Mechanical Load and Shape of the Barrel Toroid}

The Warm structure is composed of eight inner and outer radius supporting rings made of aluminum alloy beams assembled using high strength tensioning studs [4]. The $10 \mathrm{~mm}$ thick vacuum vessel is made of stainless steel $304 \mathrm{~L}$. The cold mass is 


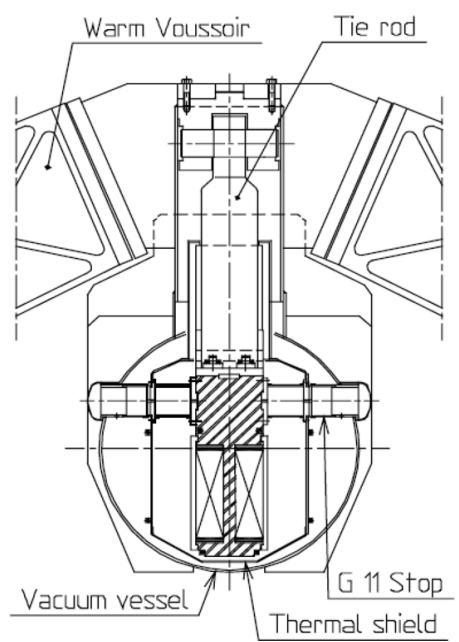

Fig. 2. Cross section of the BT cryostat showing the vacuum vessel, cold mass and the cold mass suspension system onto inner ring: tie rods and cryo-stops.

TABLE I

BARREL TOROID MAIN PARAMETERS

\begin{tabular}{ll}
\hline \hline Dimensions & $20 \mathrm{~m}$ diameter $\times 25 \mathrm{~m}$ length \\
Conductor cross section & $57 \times 12 \mathrm{~mm}^{2}$ \\
Nominal current & $20.5 \mathrm{kA}$ \\
Stored energy & $1.1 \mathrm{GJ}$ \\
Operating temperature & $4.4 \mathrm{~K}$ \\
Critical current $(94.2 \mathrm{~K}, 5 \mathrm{~T})$ & $67 \mathrm{kA}$ \\
Peak magnetic field in windings & $3.65 \mathrm{~T}$ \\
RRR (Al) & $>1000$ \\
Total weight & 1100 tons \\
\hline
\end{tabular}

suspended by eight titanium alloy Ti $5 \mathrm{Al} 2.5 \mathrm{Sn}$ tie rods, which react the resultant electromagnetic radial forces on conductors, see Fig. 2. The glass fiber reinforced epoxy resin cryogenic stops support the cold mass weight of 45 tons and accommodate the $51 \mathrm{~mm}$ total thermal shrinkage of the cold mass with a sliding system. The Barrel Toroid assembly with a weight of 1100 tons stands on stainless steel feet. The main magnetic parameters are summarized in Table I.

In order to approach the ideal cylindrical shape under self weight and service loads, the Barrel Toroid was assembled with an ellipse shape with eccentricity parameters of $\Delta y=+29 \mathrm{~mm}$ and $\Delta \mathrm{x}=-15 \mathrm{~mm}$ based on design calculations [5]. A global ANSYS model was developed at CEA and CERN (Fig. 3) to derive the deformations and resultant forces through the warm structure components and bolted connections [6]. The boundary conditions included gravitational loads, vacuum pressure, muon services load and in-plane radial magnetic forces. Survey measurements have shown an overall vertical down displacement of $27 \mathrm{~mm}$ due to the self weight, which is in good agreement with calculations. The expected additional deflection of the warm structure towards the center during powering is $2 \mathrm{~mm}$.

\section{B. Electromagnetic Force Distribution}

A 3D TOSCA model of the Barrel Toroid and calorimeter was developed (Fig. 4) to calculate the electromagnetic forces acting on the coils.

When operated alone, the Barrel Toroid is subjected to in-plane radial electromagnetic specific forces of $840 \mathrm{kN} / \mathrm{m}$

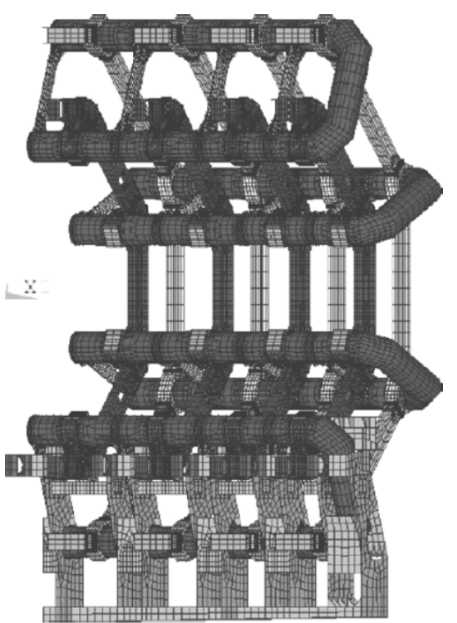

Fig. 3. Global ANSYS FEA model of the barrel toroid warm structure.

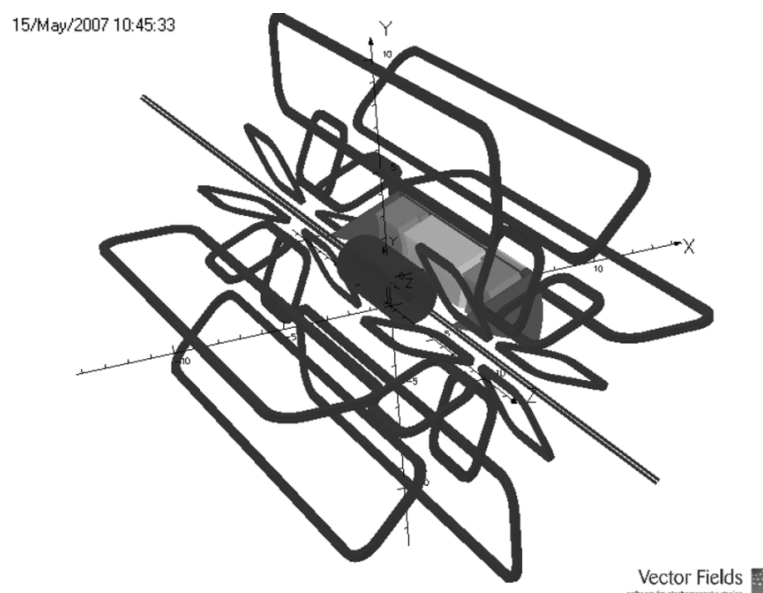

Fig. 4. View of ATLAS toroid magnet electromagnetic model.

acting on the inner coil legs and $560 \mathrm{kN} / \mathrm{m}$ on the outer ones. At nominal operation the presence of the End Cap Toroids introduces a dissymmetry in the specific force along the BT cold mass extremities. The end corner regions at inner radius are subject to a maximum specific total force of $2200 \mathrm{kN} / \mathrm{m}$ defined as $\mathrm{F}_{\mathrm{t}}=\left(\mathrm{F}_{\mathrm{r}}^{2}+\mathrm{F}_{\mathrm{z}}^{2}\right)^{1 / 2}$. The specific forces are plotted in Fig. 5 over half of the BT coil perimeter. By integrating the axial specific force $\mathrm{Fz}$, the resultant interaction force between BT and ECT is found equal to $2320 \mathrm{kN}$. The Hadronic Tile calorimeter includes a central and two end cylindrical parts which are modeled as iron annular blocks with different equivalent permeability values. Its contribution to the electromagnetic force acting on the BT conductor is limited to $15 \%$ up to 2200 $\mathrm{kN}$. This result is checked analytically with the image current method.

\section{Mechanical Load on the BT Titanium Tie Rods}

When energizing the BT coils the magnetic loads are reacted by the eight tie rods, which are part of the coil suspension system [7]. Two BT test configurations are considered for further calculations and comparison with measurements: (1) single BT test completed with Tile calorimeter in place and (2) full BT-ECT test. In order to extract the resultant forces on the tie rods an 


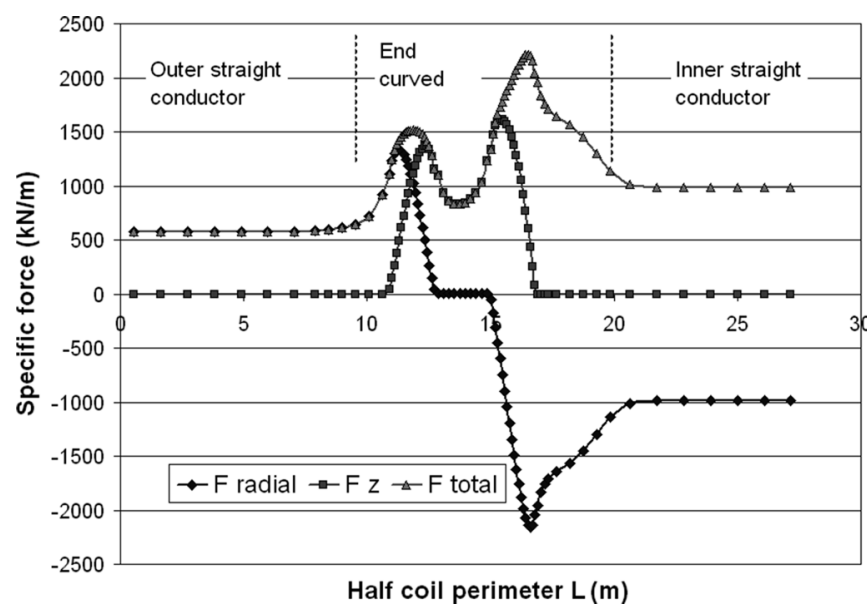

Fig. 5. Expected specific force distribution along half perimeter of BT conductors in the BT-ECT test configuration.

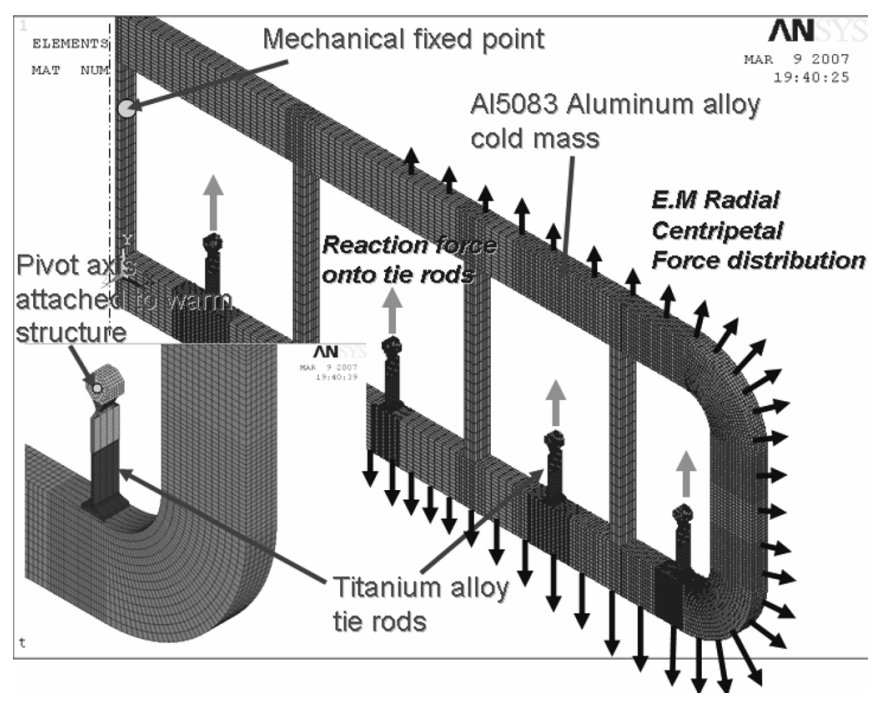

Fig. 6. FEA model of the BT cold mass for calculation of tie rods em loads.

TABLE II

BARREL TOROID MECHANICAL LOAD

\begin{tabular}{lcc}
\hline \hline Test configuration & BT + Tile & BT + ECT + Tile \\
$\begin{array}{l}\text { Expected load on tie } \\
\text { rod 1,2,3,4 (tons) }\end{array}$ & $132,115,103,60$ & $139,142,178,181$ \\
& Experimental test results & \\
$\begin{array}{l}\text { Load measurement } \\
\text { on tie rods (tons) }\end{array}$ & $114,100,98,55$ & N/A \\
$\begin{array}{l}\text { Comparison test } \\
\text { results wrt } \\
\text { prediction }\end{array}$ & $-15 \%,-15 \%,-5 \%,-9 \%$ & \\
\hline
\end{tabular}

ANSYS model of the cold mass was built (Fig. 6). The calculated distribution force is input into the FEA model, which includes the operational $10 \mathrm{MPa}$ pre-stress between windings pack and casing. The resultant expected forces are compared with the readings of the strain gages on the tie rods during the BT test with the end cap tile calorimeter in open configuration as summarized in Table II.

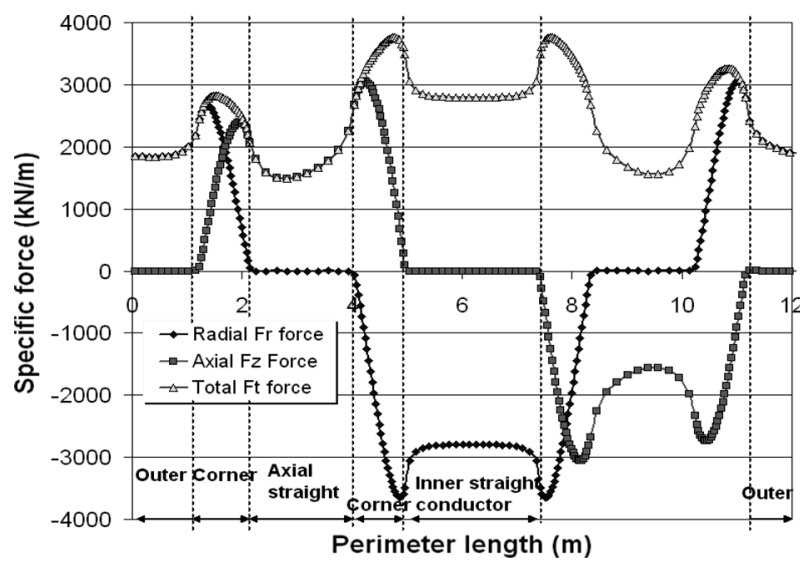

Fig. 7. Expected specific forces distribution along ECT conductors in the full system BT-ECT test configuration.

In the single BT test the current was ramped up to $14,18,19$ and $20.5 \mathrm{kA}$ corresponding to $46,77,86$ and $100 \%$ of the maximum electromagnetic load respectively. A maximum load was measured on inner tie rods up to 114 tons. The measured load levels on tie rods are up to $15 \%$ lower than predicted. The differences can be explained since part of the BT stray field is also magnetizing some surrounding iron structure, which reduces the effective radial load on the tie rods. The electromagnetic interaction between ECT and BT increases the peak total specific force on BT conductor by $55 \%$ up to $2380 \mathrm{kN} / \mathrm{m}$ with respect to the case of BT alone. This force corresponds to a maximum load on the extremity tie rod number of 181 tons. The total specific force on the ECT conductors raises up to $3800 \mathrm{kN} / \mathrm{m}$ as shown in Fig. 7.

\section{Analysis of Test Results}

The cooling down of the barrel toroid to $4.6 \mathrm{~K}$ caused a cold mass thermal shrinkage of $51 \mathrm{~mm}$ inducing a total displacement of $45 \mathrm{~mm}$ on the extremity tie rod as expected. The corresponding bending strain in the tie rod was monitored during cooling using strain gages as shown in Fig. 8 in order to check the displacements. It is observed that the tie rod feet move symmetrically around the fixed point as expected with maximum displacement of $45 \mathrm{~mm}$.

At nominal current in presence of the ECT magnets, each coil reacts a large expected centripetal force up to 1200 tons. In the last single BT test each coil has seen around $60 \%$ of this load level without the ECT coils in place. The Tile calorimeter was opened with $3.5 \mathrm{~m}$ gaps between central and end caps modules. Some full bridge cryogenic epoxy phenolic backing strain gages Vishay types WK-06-120NB were used to monitor the tensile load on each tie rod during the current ramp up. The displacement of BT warm structure was also recorded with mechanical comparators CX, CY and optical instruments on top sector and side ones.

\section{A. Displacement Measurement}

Considering the equivalent stiffness of warm structure supports it was calculated that BT would deform uniformly inwards by $d y=d x=-2 \mathrm{~mm}$. The measurements shown that the top sector raised by $d y=+3 \mathrm{~mm}$ and the side ones moved inwards 


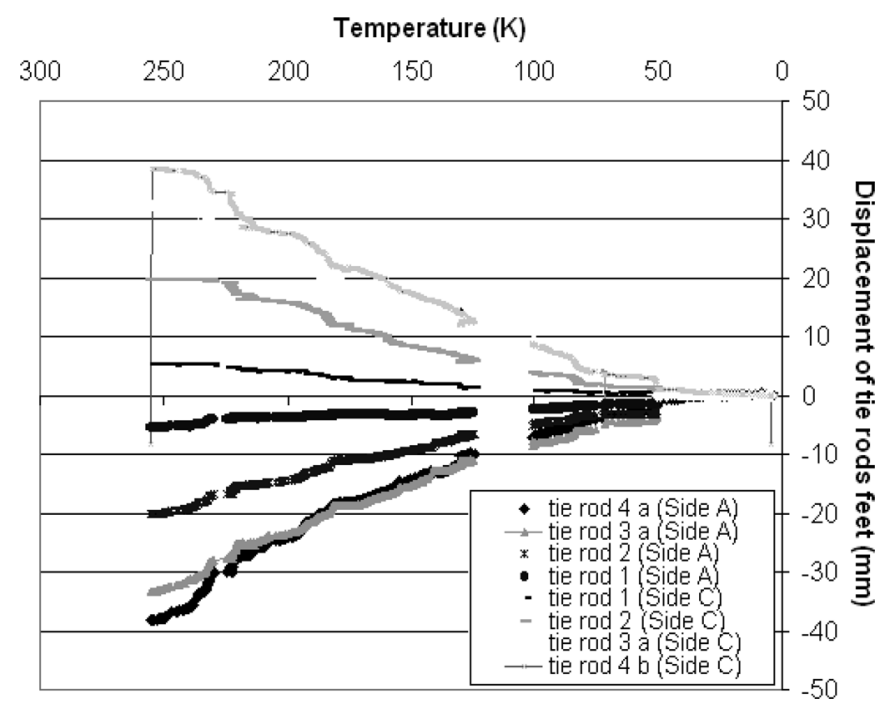

Fig. 8. Relative displacement of 8 tie rods on each coil during cool down.

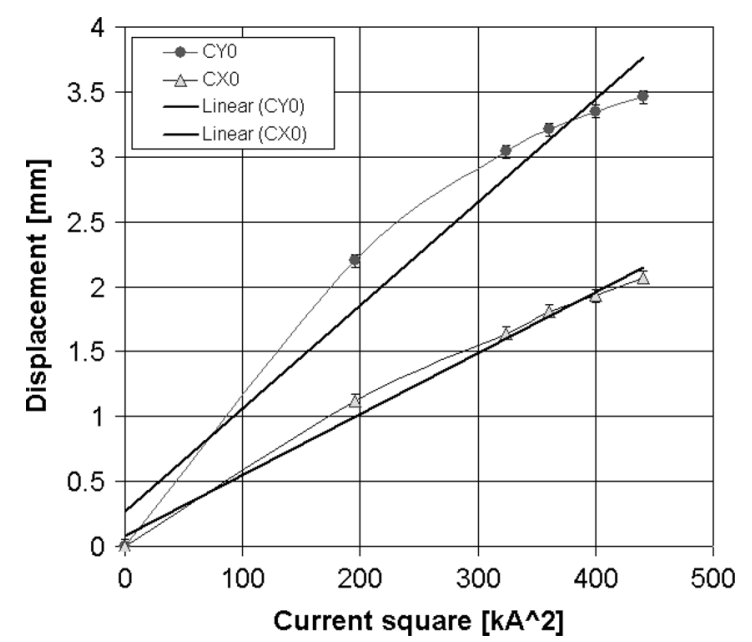

Fig. 9. Displacement measurement of BT structure during coil excitation.

by $d x=-2 \mathrm{~mm}$ (Fig. 9). This non-uniform deformation of the Barrel Toroid is characteristic of the combination of constrained displacements on bottom coils in ATLAS feet and an initial non circular assembly shape.

\section{B. Magnetic Load Measurement}

The maximum load measured on the inner central tie rods is 114 tons (Fig. 10). No hysteresis is visible, which denotes the mechanical reversibility of the BT suspension system. The analysis has shown a maximum load difference of $20 \%$ between tie rods of same category. This difference can be explained by the different azimuthal position of the BT coils.

\section{Fixed Point Measurement}

The fixed point installed on each central rib is made of fiber glass reinforced epoxy, PermaglassFE29, $50 \mathrm{~mm}$ diameter rod

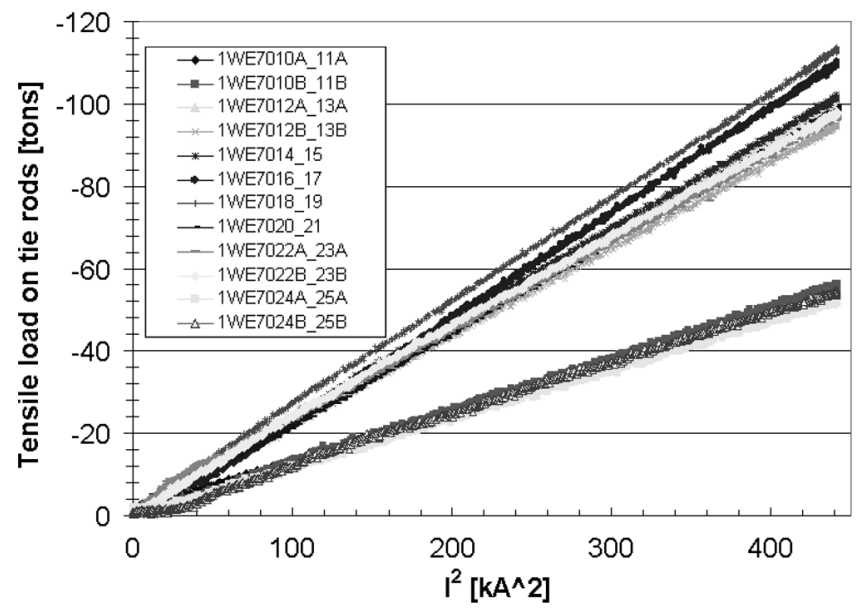

Fig. 10. Load measurement on tie rods during current ramp up to $21 \mathrm{kA}$ and followed by a fast dump.

with tensile modulus of $30 \mathrm{GPa}$ and provides a reference contact point during the $\mathrm{BT}$ cold mass thermal shrinkage. When energizing the BT coils a relative movement of $1.7 \mathrm{~mm}$ of the aluminum rib with respect to the fixed point introduces bending strain with a dynamic range of $2250 \mu \varepsilon$. This deflection is about the same as observed at the on-surface test.

\section{CONCLUSION}

An extensive testing program is performed to test and qualify of the ATLAS Barrel Toroid mechanics. The achieved load and stress levels are those of the fully assembled ATLAS Barrel toroid coil without presence of the End Cap Toroid. The good agreement found between the predictions based on a finite element model and the mechanical measurements performed during the tests demonstrates that the coil mechanical behavior is understood. The reproducibility of the evolution of the stresses on tie rods and the warm structure deformation during testing including cycling tests showed that no measurable degradation of coil mechanics occurred.

\section{REFERENCES}

[1] ATLAS Magnet collaboration, The Barrel Toroid Technical Design Report CERN, European Laboratory for Particle Physics, CERN/LHCC 97/10, 1997, ATLAS TDR.

[2] C. Berriaud, "On-surface tests of the ATLAS barrel toroid coils: Acceptance criteria and results," IEEE Trans. Appl.Supercond., vol. 16, no. 2, pp. 557-560, 2005.

[3] A. Foussat, "Assembly concept and technology on the ATLAS barrel toroid magnet," IEEE Trans. Appl. Supercond., vol. 16, no. 2, pp. 565-569, 2005, Magnet Technology MT19 Conf..

[4] Toroid Assembly_ FEA Simulations of the Assembly Phase 3 and Phase 4 internal CEA report, ref 9B 2900 T - 8000003 PA, 12/05/2003.

[5] Z. Sun, ATLAS TB Warm Structure Calculations on the Bolting Connections reference CERN report EDMS ATL-TB-EA-0005.

[6] Z. Sun, "ATLAS barrel toroid warm structure design and manufacturing," IEEE Trans. Appl. Supercond., vol. 16, no. 2, pp. 529-532, 2005, Genova.

[7] C. Mayri, "Suspension system of the barrel toroid cold mass," IEEE Trans. Appl. Supercond., vol. 16, no. 2, pp. 525-528, 2005. 